\title{
Ligature and Removal of Mesenteric Artery Aneurysm Associated to Resection of the Terminal Ileum in Patient with Endocarditis and Negative Blood Cultures
}

\author{
Ascoli Marchetti Andrea, Fabio Martinelli, Piero Rossi ${ }^{1}$, Arnaldo Ippoliti \\ Vascular Surgery, Biomedicine and Prevention Department \\ ${ }^{1}$ General Surgery, Sperimental Medicine and Surgery Department, University of Rome Tor Vergata, Italy
}

\begin{abstract}
The term mycotic aneurysm involves an infection of secondary aneurysmal wall to bacterial endocarditis. The $63 \%$ of mycotic aneurysms of the superior mesenteric artery are due to embolism from a septic endocarditis. Although most of these aneurysms is diagnosed, due to the increase in diagnostic methods, even a quarter of the superior mesenteric artery aneurysms present with rupture, intestinal ischemia and hemodynamic instability by increasing the mortality and morbidity of this disease. We report the case of a patient came to our attention for abdominal pain, suffering from bacterial endocarditis, with diagnosis of the thrombosed superior mesenteric artery aneurysm.
\end{abstract}

Keywords: Superior mesenteric artery, Endocarditis, Mycotic aneurysm, Aneurysm, Ileal bowel resection

\section{CASE Report}

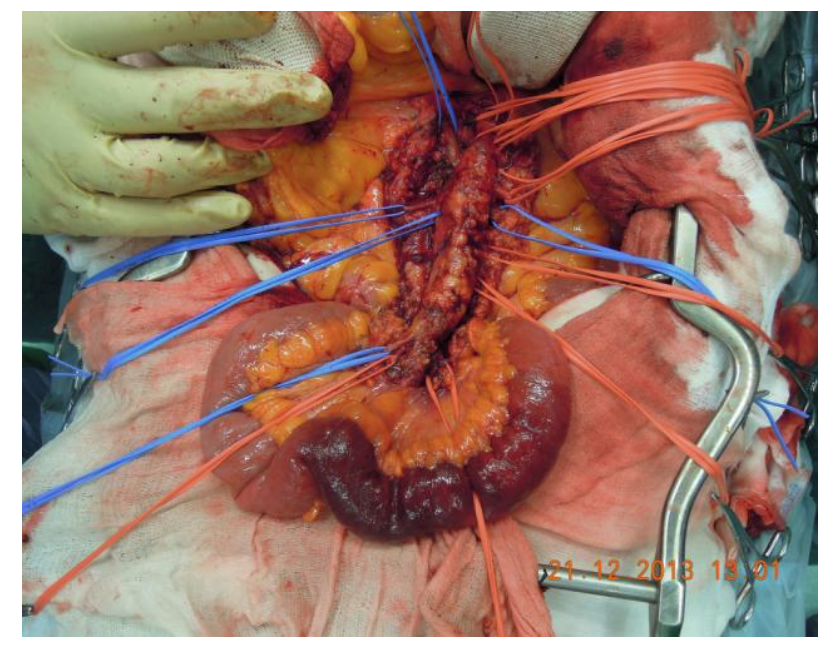

Fig 1. Intraoperative finding showing superior mesenteric artery preparation, which appears aneurysmal, and its division branches. At the end of the preparation we are appreciated ischemia of a stretch of about $10 \mathrm{~cm}$ of small intestine.

A 76 years old woman suffers from Paroxysmal Atrial Fibrillation (PAF)with known diagnosis of occlusion of the left internalcarotid artery associated with prior cerebral stroke and residual right hemiplegia and aphasia and. Suffering from aortic valve insufficiency, she had recently been subjected to replacement with a prosthetic valve implant with biological prostheses. Subsequently, history shows an episode of pericardial effusion, which resolved with pericardiocentesis $(800 \mathrm{cc}$ liquid-blood serum), and episodes of intermittent fever with negative blood cultures and urine cultures. It was later executed check-transesophageal echocardiogram that showed mobile linear images adhering to the right coronary cusp, compatible with endocarditis. For this reason, it was set antibiotic therapy with piperacillin / tazobactam and vancomycin. Following abdominal pain, the patient was submitted to Angio-CT total body, which evidence of thromboembolism of the left subclavian artery, aneurysmal dilation partially thrombosed superior mesenteric with $\mathrm{dtm} 2 \mathrm{~cm}$. 
Clinically, the patient had abdominal epi-mesogastricpain and no symptoms related to the occlusion of subclavian artery. The patient was then treated surgically by aneurysm resection, ligation of the aneurysm neck with preservation of the first jejunal branches (fig 1), the terminal ileum resection followed by ligation of the ileal artery ostium and maintenance of the first branch jejunal, not involved from the aneurysm pathology (fig 3)

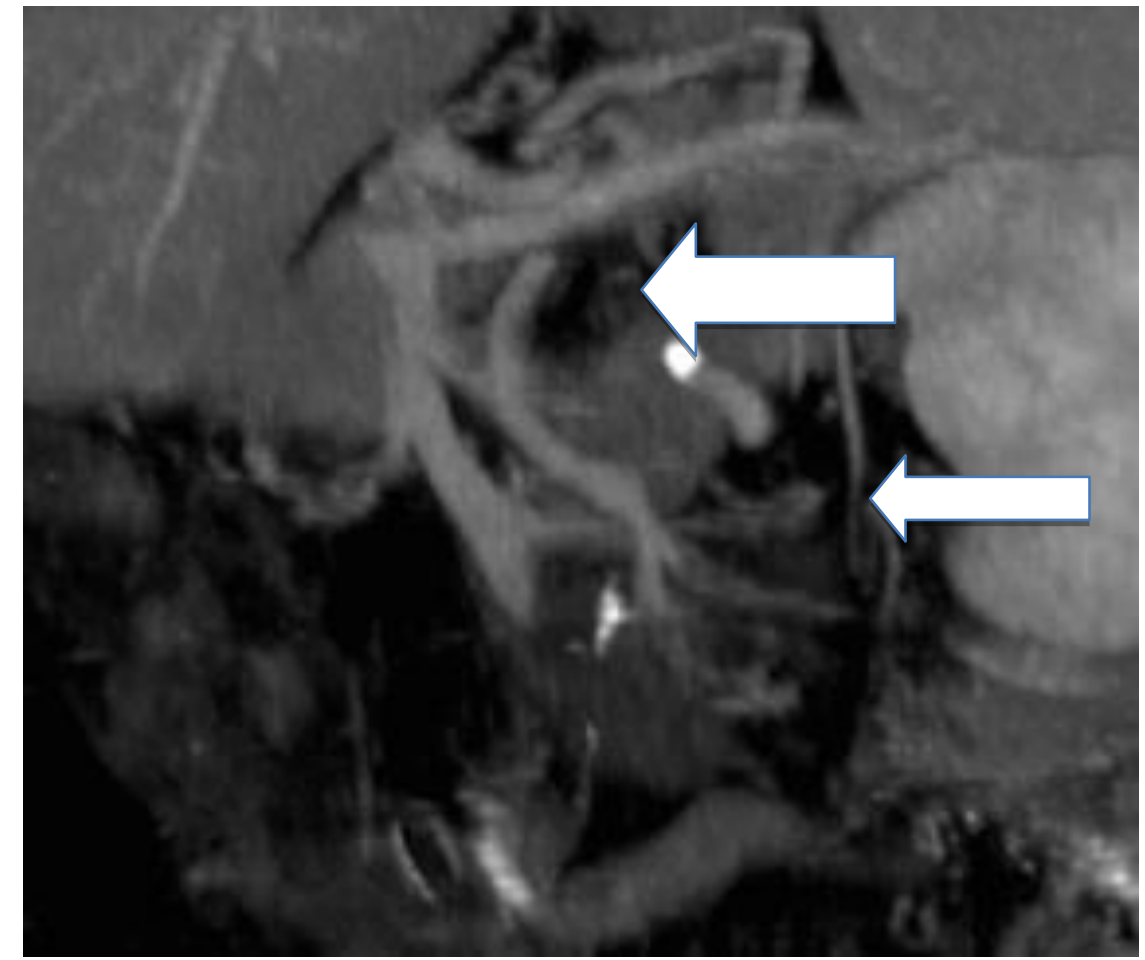

Fig 3. Postoperative Angio-Tc showing patency of the stump remaining of superior mesenteric artery (large arrow) and preserved jejunal branches (small arrow)

\section{DISCUSSION}

The aneurysm of the superior mesenteric artery is a rare disease. In the literature between 2003 and 2016 we were reported 100 cases of aneurysms of the superior mesenteric artery, but, to our knowledge, not patients with endocarditis and negative blood cultures. The septic embolization can be classified into primary, if infection occurs by contiguity from an infectious focus, and secondary if the infection also occurs at a distance from bloodborne focus $(\mathbf{1 , 2})$, where the first event is the colonization septic emboli to the vasa vasorum level. Although the aneurysm of the superior mesenteric artery is a rare complication of bacterial endocarditis, this vessel is the third most common site of all visceral aneurysms. In fact the mycotic aneurysm can occur in any vascular district (3), but can occur statistically in $31 \%$ in the abdominal aorta, in $38 \%$ in the femoral artery, the superior mesenteric artery in $8 \%$, in 5\% in the carotid artery, at $6 \%$ in iliac artery and $7 \%$ in the brachial artery $(3,4)$. The mycotic aneurysm can be treated with proximal and distal to the aneurysm ligation $(\mathbf{5})$, where the collateral circulation from the celiac trunk, from the inferior mesenteric and the hypogastric aa guarantee a good collateral flow. Also the rare inferior mesenteric artery, can be treated with simple resection (6).The use of synthetic implants or autologous substitute, with the graft interposition, are practical to perform only after having eradicated the etiologyof the infection (7). In case of saccular aneurysm, in particular in the pediatric population, is possible to perform, subsequent to the ligation of the aneurysm and its resection, the aorto mesenteric bypass to ensure perfusion of the districts to downstream of the aneurysm. In our case we decided to remove the aneurysm, opting for ligation of the upstream vessel, to ensure a more complete eradication of infected material, and bowel resection to prevent ischemic complications during surgery was performed by evaluating the involved bowel. The intraoperative finding of thrombosed aneurysm and fig 2 intestinal ischemia, justified the symptoms complained by the patient, which necessitated the intervention in emergency regime. The postoperative course was uneventful. 


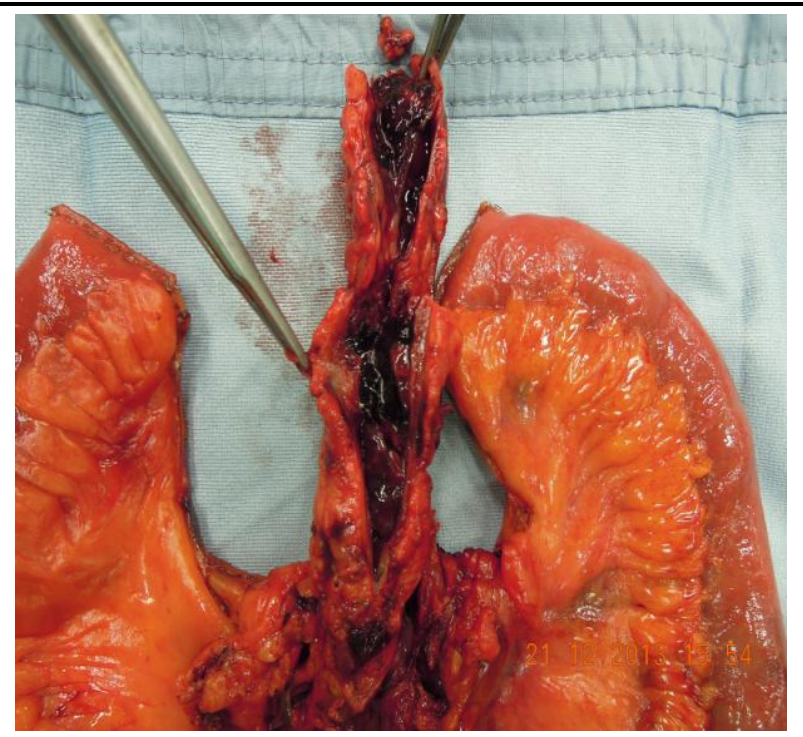

Fig 2. Intraoperative finding showing the aneurysm sac after longitudinal section of the sac longitudinally. Note the recent thrombosis

\section{CONCLUSIONS}

Although the mycotic aneurysm is a rare occurrence, it can be said that its evolution towards a serious complication is high with a significant mortality rate. Despite the progress of still $25 \%$ of imaging methods of this pathology is diagnosed only after the occurrence of a complication. The aneurysm resection and intestinal segment involved, prevents aneurysm rupture and / or the evolution of intestinal ischemia.

\section{REFERENCES}

[1] Kirkwood ML1, Knowles M, Modrall JG, Valentine RJMycotic inferior mesenteric artery aneurysm secondary to native valve endocarditis caused by coagulase-negative Staphylococcus Ann Vasc Surg. 2014 Jul;28(5):1312

[2] Chai HT, Tan BL, Yen HT, Chen MC. Infective endocarditis caused by Streptococcus bovis complicated by a superior mesenteric arterymycoticaneurysm and systemic septic emboli in a patient with colon diverticulitis. Int J Infect Dis. 2010 Sep;14 Suppl 3:e317-8.

[3] Lorelli DR, Cambria RA, Seabrook GR, Towne JB. Diagnosis and management of aneurysms involving the superior mesenteric artery and its branches--a report of four cases. Vasc Endovasc Surg 2003 Jan-Feb;37(1):59-66.

[4] Ippoliti A, Ascoli Marchetti A, Pratesi G, Di Giulio L, Battistini M, Patacconi D. Aneurismi splancnici e dell'arteria renale. In:. Chirurgia vascolare ed endovascolare di Guido Regina. vol. 1, p. 155-162, Italy, PADOVA:PICCIN 2014

[5] de Troia A, Mottini F, Biasi L, Azzarone M, Tecchio T, Salcuni P.Superior Mesenteric ArteryAneurysm Caused by Aortic Valve Endocarditis: The Case Report and Review of the Literature.Vasc Endovascular Surg. 2016 Feb;50(2):88-93

[6] Sriussadaporn S, Prichayudh S, Sriussadaporn S, Kritayakirana K, Pak-art R.Successful management of a superior mesenteric artery aneurysm by ligation: a case report. J Med Assoc Thai2006 Nov;89(11):1965-9.

[7] Rega FR, Nevelsteen A, Peetermans WE, Herregods MC, Flameng W, Herijgers P. Simultaneous valve replacement and venous patch repair of superior mesenteric arteryaneurysm due to infective endocarditis: a case report. Heart Surg Forum. 2006;9(5):E741-3. 